\title{
Ureteric foreign body following previous aorto-iliac graft
}

\author{
Vitor da Silva, MD; Jonathan Taylor, $M D^{+}$ \\ "Division of Urology, The Ottawa Hospital, University of Ottawa, Ottawa, ON; 'Division of Urology, Chinook Regional Hospital, Lethbridge, AB
}

Cite as: Can Urol Assoc J 2015;9(5-6):E300-1. http://dx.doi.org/10.5489/cuaj.2368 Published online May 13, 2015.

\section{Abstract}

Foreign bodies in the ureter are extremely rare. We report a case of an 82-year-old man who, after an incidental presentation with asymptomatic hydroureteronephrosis, had an intraureteric foreign body. The patient was managed with atraumatic endoscopic extraction of the specimen.

\section{Introduction}

Foreign bodies in the ureter are extremely rare. Reports of ureteric foreign bodies have included retained stents, renal artery angio-embolization coils, surgical clips from pyeloplasty, traumatic shrapnel, and foreign bodies introduced per urethra. ${ }^{1-3}$ There is one report of a ureteric plastic toothpick, which eroded from the gastrointestinal tract following accidental ingestion 15 years prior. ${ }^{4}$ Furthermore, there have been several reports of hydronephrosis following vascular grafting. ${ }^{5}$ We report what we believe is the first case of an obstructing ureteric foreign body following previous aortoiliac grafting.

\section{Case report}

An 82-year-old man was referred for urologic assessment following an incidental finding of new right-sided hydroureteronephrosis seen on an abdominal computed tomography (CT) scan performed for unrelated reasons (Fig. 1, Fig. 2). No calculus was identified on the non-contrast phase of the $\mathrm{CT}$. A urogram phase was not performed and the patient was asymptomatic. He denied any previous episodes of flank pain or gross hematuria.

His medical history includes hypertension, type II diabetes, coronary artery disease (with previous 5-vessel coronary artery bypass graft surgery in 2004), chronic kidney disease (baseline estimated glomerular filtration rate [eGFR] 40, baseline creatinine $\sim 130$ ), and bilateral iliac aneurysms (with aortobiiliac grafting in 2008).

Physical examination was unremarkable. Urine culture and cytology were negative. Bloodwork revealed an eGFR of 33 and creatinine of 165 . A diuretic renal scan confirmed right hydroureteronephrosis to the pelvic ureter and delayed excretion in keeping with a ureteric obstruction. He was brought to undergo a right retrograde pyelogram with possible diagnostic ureteroscopy for further investigation.

The retrograde pyelogram demonstrated a narrowed segment of lumen in the distal right ureter (Fig. 3). Ureteroscopy at this level revealed what appeared to be a soft-tissue mass filling the entire ureteric lumen. Using grasping forceps, we attempted a biopsy of the lesion, though surprisingly it was freely removed in toto. Closer inspection of the specimen revealed two $1-\mathrm{cm}^{2}$ Gortex pieces attached by a prolene suture (Fig. 4). A double-J stent was subsequently placed.

\section{Discussion}

A recent review of the literature found 27 papers describing hydronephrosis following vascular graft placement. ${ }^{5}$ All of these reported ureteric stenosis, external compression or retroperitoneal fibrosis as the cause for ureteric obstruction. No ureteric foreign bodies following vascular grafting have been previously described.

In our case, the anatomic location and material found within the lumen of the ureter were consistent with foreign body erosion into the ureter from the aortoiliac graft. It appeared to be a pledget, commonly used during vascular grafting. A review of the operative report did not reveal any intraoperative complications to suggest a ureteric injury at the time of graft placement.

\section{Conclusion}

We believe this is the first reported case of an intraureteric foreign body causing asymptomatic hydroureteronephro- 


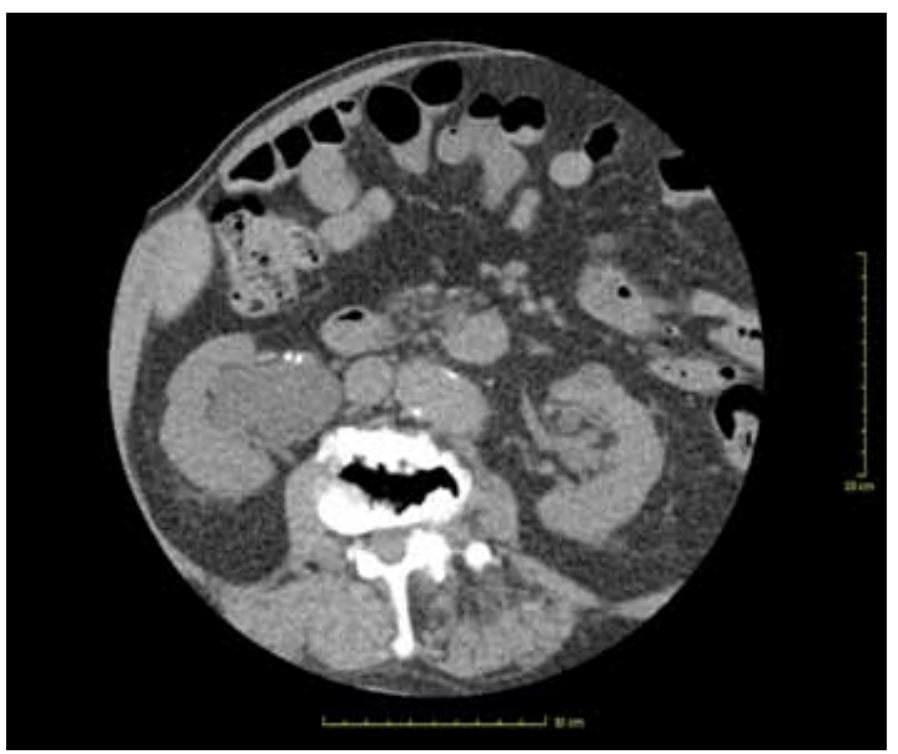

Fig. 1. Non-contrast computed tomography abdo-pelvis demonstrating proximal hydroureteral nephrosis.

sis following aortoiliac graft placement. We were able to successfully manage this endoscopically, with atraumatic extraction of the specimen. Whether the patient will have a residual stenosis or long-term obstruction remains to be seen.

Competing interests: The authors declare no competing financial or personal interests.

This paper has been peer-reviewed.

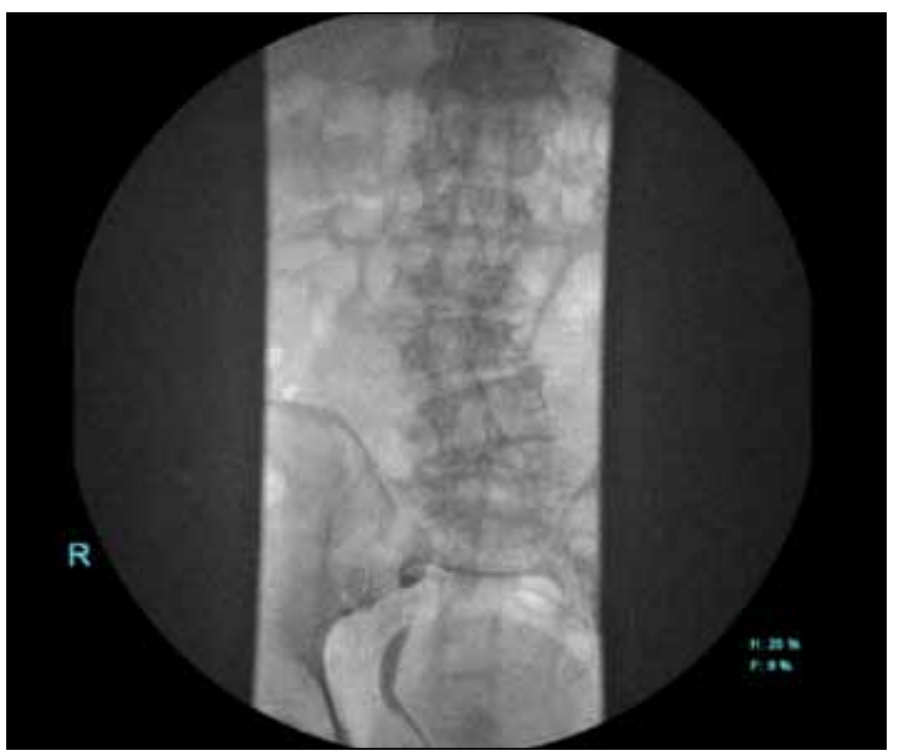

Fig. 3. Retrograde pyelogram demonstrating stenotic segment of the distal ureter.

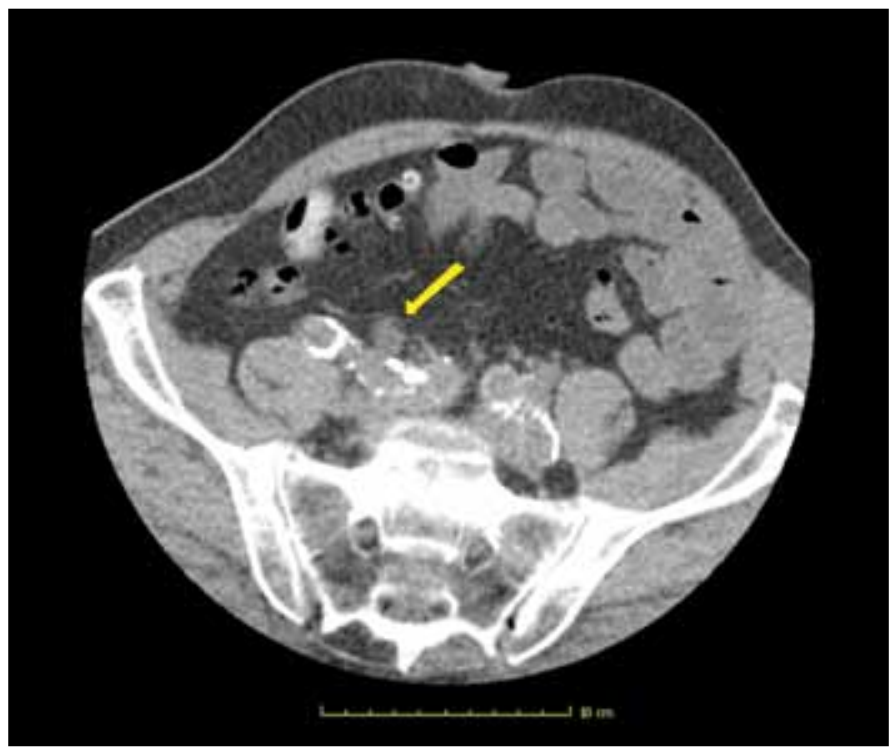

Fig. 2. Non-contrast computed tomography of the abdomen and pelvis demonstrating a dilated ureter at the level of the obstruction. Note the anatomic relationship to the surrounding vasculature.

\section{References}

1. van Ophoven A, deKernion JB. Clinical management of foreign bodies of the genitourinary tract. J Urol 2000;164:274-87. http://dx.doi.org/10.1016/S0022-5347(05)67342-9

2. Brusky JP, Tran VQ. Resorbable clip migration resulting in ureteral obstruction and sepsis after laparoscopic pyeloplasty. J Endourol 2010;24:1563-4. http://dx.doi.org/10.1089/end.2010.0068

3. Rajesparan K, Partridge W, Taha N, et al. Early migration and ureteric obstruction of an embolisation coil used to treat massive haemorrhage following percutaneous nephrolithotomy. Cardiovasc Intervent Radiol 2011;34:868-72. http://dx.doi.org/10.1007/s00270-011-0178-y

4. Plavcan WG, McWilliams WA. Toothpick obstruction of the ureter. J Urol 1988;139:114-5.

5. Borges LL, Torricelli FCM, Ebaid GX, et al. Urological complication following aortoiliac graft: Case report and review of the literature. Sao Paulo Med J 2010;128:174-6. http://dx.doi.org/10.1590/S151631802010000300010

Correspondence: Dr. Jonathan Taylor, Division of Urology, Chinook Regional Hospital, 100-1410 Mayor Magrath Dr S., Lethbridge, AB TIK 2R3; ist123@shaw.ca

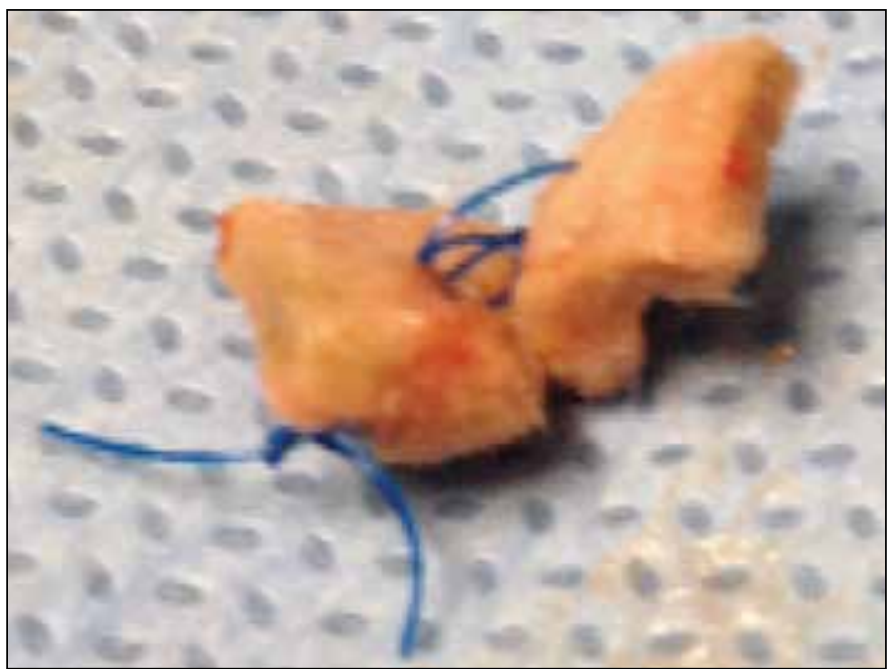

Fig. 4. Foreign body removed from ureter. 